Gold seed fiducials in analysis of linear and rotational displacement of the prostate bed

\title{
Ålander, Elisa
}

2014

Ålander , E , Visapää , H , Kouri , M O , Keyriläinen , J , Saarilahti , K \& Tenhunen , M 2014 , ' Gold seed fiducials in analysis of linear and rotational displacement of the prostate bed ' , Radiotherapy and Oncology, vol. 110 , no. 2 , pp. 256-260 . https://doi.org/10.1016/j.radonc.2013.10.037

http://hdl.handle.net/10138/45199

https://doi.org/10.1016/j.radonc.2013.10.037

submittedVersion

Downloaded from Helda, University of Helsinki institutional repository.

This is an electronic reprint of the original article.

This reprint may differ from the original in pagination and typographic detail.

Please cite the original version. 
Original article

\title{
Gold seed fiducials in analysis of linear and rotational displacement of the prostate bed
}

\author{
Elisa Ålander*, Harri Visapää, Mauri Kouri, Jani Keyriläinen, Kauko Saarilahti, Mikko Tenhunen \\ Helsinki University Central Hospital, Department of Oncology, Finland
}

\section{A R T I C L E I N F O}

\section{Article history:}

Received 30 July 2013

Received in revised form 25 October 2013

Accepted 26 October 2013

Available online $\mathrm{xxxx}$

\section{Keywords:}

Prostate bed motion

Gold seed fiducials

Rotation of the prostate bed

Margin

Prostate cancer

\begin{abstract}
A B S T R A C T
Background and purpose: This study aimed to investigate the magnitude of interfraction prostate bed motion during radiotherapy using both the implanted gold seed fiducials and the soft tissue registration and to define reasonable planning target volume (PTV) margins for different localization methods. Material and methods: Thirteen prostatectomized prostate cancer patients, after implanting four gold seed fiducials into their prostate bed, were imaged daily using a pretreatment cone-beam computed tomography (CBCT). Linear and the rotational prostate bed motion (PBM) was measured for 466 CBCTs.

Results: The linear PBM mean and standard deviation values in millimeters are $0.0 \pm 0.5,0.7 \pm 2.1$ and $0.8 \pm 1.6$ in the LR, SI and AP axes, respectively. In $20 \%$ of the fractions the rotation of the prostate bed in sagittal plane exceeds $\pm 6^{\circ}$ and in $5 \%$ it exceeds $\pm 10^{\circ}$ from the position on the planning CT. In the transversal and coronal planes $1 \%$ and $2 \%$ of it exceeds $\pm 6^{\circ}$. The PTV margins are $2.4,6.5$ and $6.6 \mathrm{~mm}$ in the LR, SI and AP axes, respectively, if imaging is performed for the first three treatment fractions.

Conclusion: The linear PBM is largest in the SI and AP axis, whereas the rotation is largest in the sagittal plane. Bone localization during the first three treatment fractions can reduce PTV margins by $52 \%, 18 \%$ and $10 \%$ in the LR, SI and AP axes, respectively, whereas in daily CBCT the use of the gold seed fiducials seems profitable.
\end{abstract}

(C) 2013 Elsevier Ireland Ltd. All rights reserved. Radiotherapy and Oncology xxx (2013) xxx-xxx
Radiotherapy as adjuvant and salvage therapy following radical prostatectomy improves progression free survival and has been increasingly applied [1-3]. Recent studies of the movement of the prostate bed have shown that in a large proportion of the treatment fractions total position error (TPE) exceeds $5 \mathrm{~mm} \mathrm{[4-6].}$

Conformal radiation, such as intensity-modulated radiation therapy (IMRT) and volumetric modulated arc therapy (VMAT), has been shown to reduce gastrointestinal and genitourinary toxicity compared to conventional radiotherapy by creating steep dose falloffs along strict margins [7,8]. A desirable dose coverage to the target while sparing adjacent critical normal tissues requires that the position of the prostate bed is known precisely. Conebeam computed tomography ( $\mathrm{CBCT}$ ) with a high contrast 3D visualization of the anatomy allows localization based on soft tissues. In 2D X-ray imaging system this is not possible but gold seed fiducials placed to the prostate bed facilitate the prostate bed localization. Schiffner et al. [4] introduced a method for the prostate bed localization by gold seed fiducials and showed that there was no significant migration of the gold seeds during the treatment period. In another study Williams et al. [9] found placing the gold

\footnotetext{
* Corresponding author. Address: Helsinki University Central Hospital, Department of Oncology, Haartmaninkatu 4, PL 180, 00029 Hus, Finland.

E-mail address: elisa.alander@gmail.com (E. Ålander).
}

seeds difficult since anatomic landmarks are distorted and there is an absence of substantial tissue to hold the markers in place.

Prostate bed motion (PBM) has been examined thoroughly by ultrasound-based technologies [10,11], by СВCT in combination of rectal wall or surgical clips localization [6,12-14], by electronic portal imaging device (EPID) and gold seed fiducials localization [4], and by orthogonal kV images together with radiofrequency beacons or surgical clips localization $[5,15]$. In this study we investigate the prostate bed localization difference between soft tissue registration and gold seed fiducial localization. In addition to the linear PBM also the rotational movements are measured. We also derive desirable planning target volume (PTV) margins to the cases where image-guided localization is performed using an analysis of bony anatomy landmarks, gold seed fiducial markers or soft tissue registration and in a situation where image-guidance is applied more sparsely and patient set-up is done according to patient skin marks.

\section{Materials and methods}

\section{Patients}

The study population consists of thirteen prostate cancer patients 55-74 years of age who were prostatectomized between the years of 2000 and 2011 . Because of a rising postoperative pros- 
tate specific antigen they received post prostatectomy radiotherapy (either $2 \mathrm{~Gy} \times 33=66 \mathrm{~Gy}$ or $1.8 \mathrm{~Gy} \times 37=66.6 \mathrm{~Gy}$ ) to the prostate bed during 2012 .

\section{Implantation of the gold seed fiducials}

Two weeks before treatment period four gold seed fiducials were implanted into the prostate bed. Placement of the gold seeds was done by an urologist in a transrectal ultrasound guidance. Two fiducials were placed at the urethrovesical junction, one to the right and one to the left side, and two fiducials were placed in the area of the base of seminal vesicles, one to the right and one to the left side. The approximate places of the fiducials are shown in Fig. 1. The fiducials were utilized as a supporting method for the treatment localization.

\section{Measurement of total position error, set-up error and prostate bed motion}

Patients were treated with the Elekta Axesse linear accelerator using VMAT. Initial setup was done according to skin marks (SM). Daily CBCTs were applied before treatment and final prostate bed positioning was defined according to them. Absorbed dose of a prostate CBCT protocol was $18 \mathrm{mGy}$. In total, 466 pretreatment CBCTs were analyzed and compared to reference CTs. At first, localization was done according to bones and corrections were performed in three directions relative to isocenter; left-right (LR), superior-inferior (SI) and anterior-posterior (AP). Performed corrections represent set-up error (SUE). Localization was executed also along the gold seed fiducials. Positions of the fiducials were compared to planning $\mathrm{CT}$ and the difference in location represents TPE. The TPE includes patient SUE and PBM hence PBM was calculated as a difference between TPE and SUE. The X-ray volume imaging (XVI) version R4.5 CBCT imaging system includes an automatic soft tissue registration where the registration was performed
Translational

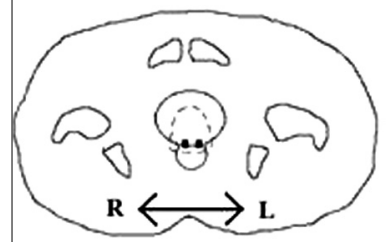

Left-Right axes

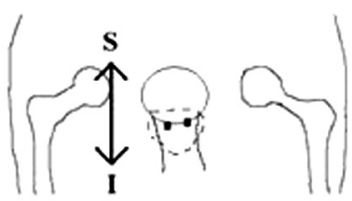

Superior-Inferior axes

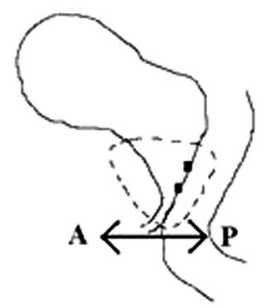

Anterior-Posterior axes
Rotational

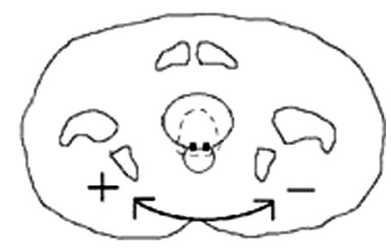

Transversal plane

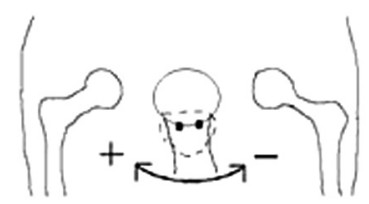

Coronal plane

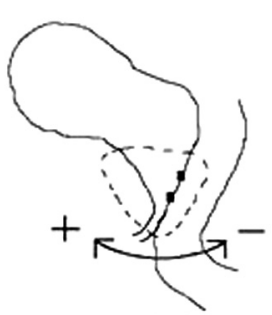

Sagittal plane
Fig. 1. The translational and the rotational prostate bed motion directions. For clarity, only two fiducials are shown. with the volume of PTV $+2 \mathrm{~cm}$ margin. That registration gives also the TPE and hence the PBM can be calculated based on two different localization methods. Mean $(\bar{m})$ and standard deviation (SD) values were determined for TPE, SUE and PBM.

There is always a possibility that fiducials migrate and this was measured by comparing the distances of the fiducials at the beginning and at the end of the treatment period and once per week in between. The mean $(\bar{m})$ and the SD values were calculated for the changes in inter-fiducial distances for all patients and all seeds in total of 110 fractions.

\section{Measurement of rotation of the prostate bed}

In addition to linear PBM the prostate bed rotation (PBR) was examined by analyzing the rotational position error of gold seed fiducials. The PBR was determined using the center of the prostate bed as a center of rotation and then comparing the fiducial rotational position of the CBCTs to that of the reference CT. Rotation was determined in three different planes, i.e., transversal, coronal and sagittal, which are presented in Fig. 1. The analysis included ten first treatment fractions of each patient leading to 130 fractions in total.

\section{CTV-PTV margins}

PTV margins were defined in three separate directions, i.e., LR, SI and AP axes, from the entire patient data using the methods represented by van Herk et al. [16]. Requiring that $95 \%$ of the patients achieve minimum dose of $95 \%$ from prescribed dose to the CTV the margin calculation was performed by an equation of

$1.96 \sum+0.7 \sigma$,

where $\Sigma$ represents systematic error and $\sigma$ random error. Margins calculated from systematic and random errors of TPE represent margins which are needed if the set up is based only on patient SM. If imaging is performed on a daily basis and alignment is done by bone localization (BL), which gives the adequate margins for $2 \mathrm{D}$ imaging guidance without gold seeds, margins can be calculated from systematic and random errors of the PBM. In third case imaging is done during the first three treatment fractions, the position of systematic displacement of the prostate bed is defined according to $\mathrm{BL}$ and marked on the skin, and the rest of the fractions are treated according to the offset SM. The PTV margins should then take into account the difference between SUEs (the SUE difference), which are counted from the first three fractions and from the entire treatment period, systematic error of the PBM and random errors of the SUE and PBM. The equation of the PTV margin can now be written as

$1.96 \sqrt{\sum_{\mathrm{SUE}_{\text {difference }}}+\sum_{\mathrm{PBM}^{2}}}+0.7 \sqrt{\sigma_{\mathrm{SUE}^{2}}+\sigma_{\mathrm{PBM}^{2}}}$.

\section{Results}

Total position error, set-up error and prostate bed motion

The values of $\bar{m}$ and SD determined to TPE, SUE and PBM in LR, SI and AP directions are listed in Table 1. For the PBM $\bar{m} \pm$ SD in millimeters are $0.0 \pm 0.5,0.7 \pm 2.1$ and $0.8 \pm 1.6$ when PBM is measured based on the position of gold seed fiducials and $-0.1 \pm 0.4$, $0.4 \pm 0.9$ and $0.1 \pm 0.5$ when motion is defined by soft tissue registration. The difference of the PBM between these different localization methods is described by a $p$-value which is shown in Table 1. The $p$-value was less than 0.05 in LR and AP axes while in SI axes it was found to be 0.067 . The linear PBM counted from gold seed fiducials is shown in Fig. 2. Fig. 2 represents the average and max- 
Table 1

Mean and standard deviation values $[\mathrm{mm}]$ and $p$-values for the difference between prostate bed motion defined by gold seed fiducials and soft tissue registration.

\begin{tabular}{|c|c|c|c|c|c|}
\hline & TPE & SUE & PBM (fiducials) & PBM (soft tissue) & $p$-Value \\
\hline $\mathrm{LR}(\bar{m} \pm \mathrm{SD})$ & $1.2 \pm 1.8$ & $1.2 \pm 1.8$ & $0.0 \pm 0.5$ & $-0.1 \pm 0.4$ & 0.027 \\
\hline $\mathrm{SI}(\bar{m} \pm \mathrm{SD})$ & $0.3 \pm 2.9$ & $-0.5 \pm 2.0$ & $0.7 \pm 2.1$ & $0.4 \pm 0.9$ & 0.067 \\
\hline $\mathrm{AP}(\bar{m} \pm \mathrm{SD})$ & $0.4 \pm 2.7$ & $-0.2 \pm 2.2$ & $0.8 \pm 1.6$ & $0.1 \pm 0.5$ & 0.000 \\
\hline
\end{tabular}

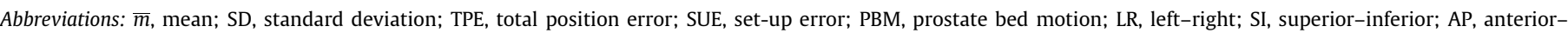
posterior.

imum values of the PBM for each patient in LR, SI and AP axes, respectively. There was no significant migration of the gold seeds. The mean $(\bar{m})$ and the SD values of the changes in inter-fiducials distances over all seeds and all patients were found to be $0.0 \mathrm{~mm}$ and $1.1 \mathrm{~mm}$, respectively. The range of the changes in inter-fiducial distances in millimeters was $[-4.0 ; 5.7]$ where the negative value corresponds to the change of smaller distance and the positive value corresponds to the change of larger distance than the average.

\section{Rotation of the prostate bed}

The values of $\bar{m}$ and SD for the PBR are shown in Table 2. Fig. 2 represents the maximum deflections of the PBR for each patient. Mean values of the PBR are close to zero degrees in all the rotation directions, whereas there is more variation in SD values. The standard deviation (SD) on a sagittal plane is $\pm 3.6^{\circ}$ which is the largest value.

Table 2 also represents the distribution of rotation values. The percentages of treatment fractions where PBR is more or equal to $\pm 6^{\circ}$ are $1 \%, 2 \%$ and $20 \%$ in the transversal, coronal and sagittal planes, respectively. The PBR in sagittal plane is over or equal to $\pm 10^{\circ}$ in $5 \%$ of the treatment fractions.

\section{CTV-PTV margins}

Table 3 shows the results of the calculated systematic and random errors of the TPE, SUE and PBM and calculated PTV margins. The PBM margins of $1.4,5.9$ and $5.9 \mathrm{~mm}$ in the LR, SI and AP axes, respectively, are needed in daily BL. The BL on the first three treatment fractions and the offset SM localization on the rest
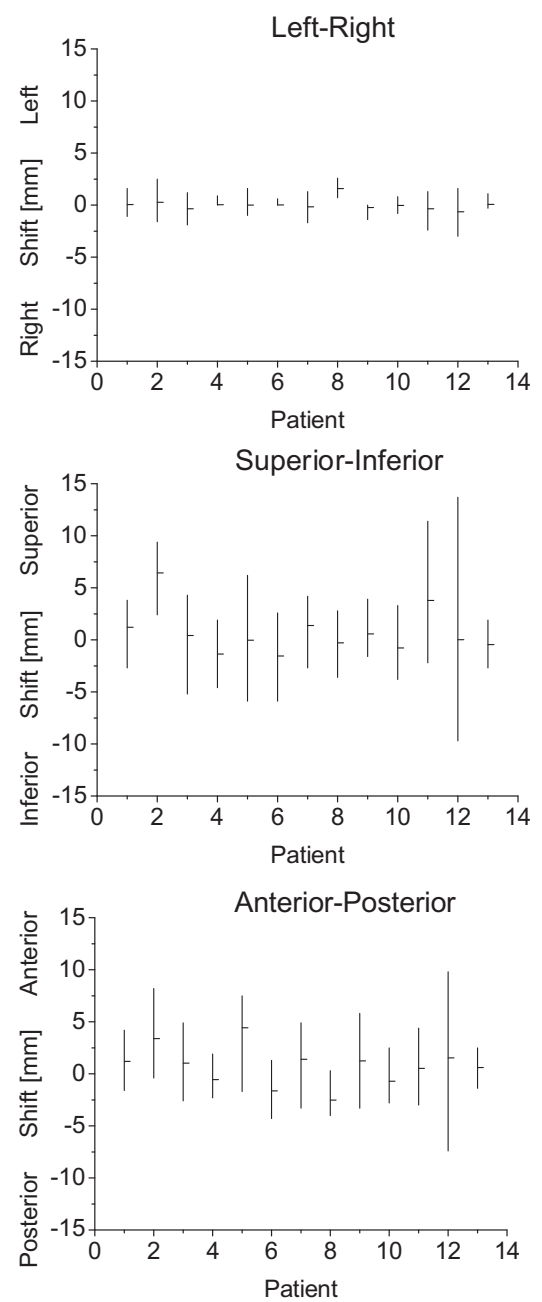
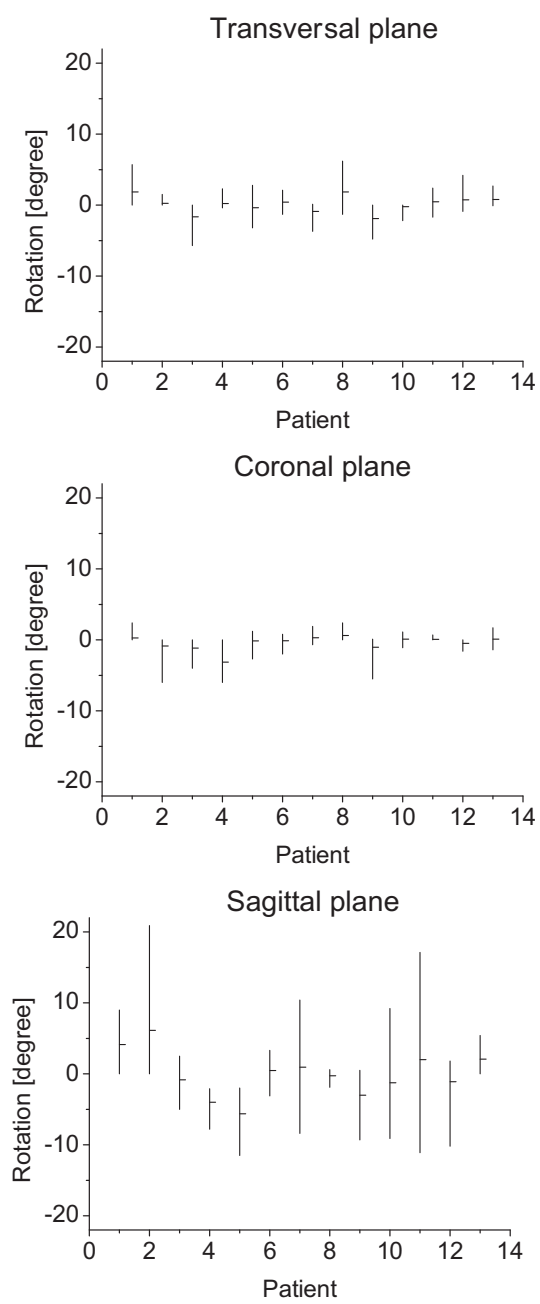

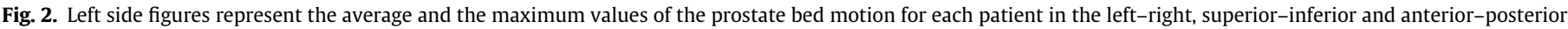
axes, respectively. The rotational values are shown on the right side in the transversal, coronal and sagittal plane, respectively. 
Table 2

Rotation of the prostate bed and percentages of treatment fractions where prostate bed rotation is less or equal to $\pm 4^{\circ}$ or larger or equal to $\pm 6^{\circ}, \pm 8^{\circ}$ and $\pm 10^{\circ}$ in the transversal, coronal and sagittal planes.

\begin{tabular}{llll}
\hline & Transversal plane & Coronal plane & Sagittal plane \\
\hline $\bar{m} \pm \mathrm{SD}$ & $0.1^{\circ} \pm 1.4^{\circ}$ & $-0.4^{\circ} \pm 1.1^{\circ}$ & $0.0^{\circ} \pm 3.6^{\circ}$ \\
$\geqslant \pm 4^{\circ}$ & $7 \%$ & $5 \%$ & $33 \%$ \\
$\geqslant \pm 6^{\circ}$ & $1 \%$ & $2 \%$ & $20 \%$ \\
$\geqslant \pm 8^{\circ}$ & $0 \%$ & $0 \%$ & $13 \%$ \\
$\geqslant \pm 10^{\circ}$ & $0 \%$ & $0 \%$ & $5 \%$ \\
\hline
\end{tabular}

Abbreviations: $\bar{m}$, mean; SD, standard deviation.

of the fractions require margins of 2.4, 6.5 and $6.6 \mathrm{~mm}$ in the LR, SI and AP axes, respectively.

\section{Discussion}

The values of $\bar{m}$ and SD show that the systematic part of the TPE is mainly caused by the SUE while the PBM consists only little of the systematic error and thus the motion is mostly caused by the random error. The linear PBM is smallest in LR direction and larger in SI and AP axes. Fig. 2 shows that some of the patients have more remarkable movements in their prostate bed while with the others the movement is minor. Gill et al. [17] noticed the same result in their study of adaptive post-prostatectomy radiotherapy. The linear PBM determined by the gold seed fiducials was performed also by Schiffner et al. [4]. They used orthogonal EPID images together with mainly two fiducials for the localization, so that three dimensional information from the PBM was not gathered there.

This is the first study to compare PBM analyzed by both gold seed fiducials and soft tissue registration. Different localization methods give unequal results. The motion calculated from the gold seeds, which are implanted near to the posterior wall of the prostate bed, delineates mainly the movement of the rectal wall of the prostate bed while soft tissue registration defines the average movement of the prostate bed and other soft tissues. Rectal wall is aimed to be highly protected from an unnecessary dose and, furthermore, local recurrences and metastases are more probable to arise near the posterior wall of the prostate bed $[18,19]$. Hence, it is clinically important to know the precise location of the posterior wall. Gold seed fiducials seem to enable more accurate localization of the prostate bed posteriorly. Ost et al. [6] used anterior rectal wall to match posterior wall of the prostate bed of the CBCT with the $\mathrm{CT}$ and the matching was done in collaboration with a radiation oncologist and a therapist. From a practical point of view, attending both observers in every treatment fraction is probably not possible at every hospital, whereas the gold seed implantation enables the localization of the prostate bed by therapists alone.

Fig. 2 shows that as in linear PBM the PBR seems to be relatively large on some of the patients and smaller with others. Interestingly, considering all the patients PBR in transversal and coronal planes is mainly less than $\pm 6^{\circ}$ but in the sagittal plane $20 \%$ of the treatment fractions exceeds it. If we assume the diameter of the target to be $7 \mathrm{~cm}, \pm 6^{\circ}$ rotation removes the target border by $3.7 \mathrm{~mm}$ cranially or caudally. In $5 \%$ of the treatment fractions the PBR in sagittal plane was over $\pm 10^{\circ}$ which means $6.2 \mathrm{~mm}$ shift in cranial or caudal direction. In the newest isocentric linear accelerators and Cyberknife system it is possible to utilize tilting couch angles with a range of $\pm 6^{\circ}$ to correct the rotation of the treatment targets. The angular range of the linac treatment robotic couch rotation is typically $\pm 3^{\circ}$. Nevertheless, the rotation less than $\pm 4^{\circ}$ moves the prostate bed by $2.4 \mathrm{~mm}$ in cranial or caudal direction and the correction may be unnecessary. The rotations larger than $\pm 6^{\circ}$ should be corrected in another way, like using an adaptive planning method. Based on our findings, there seems to be no direct dependence between rotational and linear motion of the prostate bed.

The CBCT and the BL on the first three treatment fractions can reduce CTV-PTV margins by $52 \%, 18 \%$ and $10 \%$ in the LR, SI and AP axes, respectively, as compared to the localization with SM. Daily BL does not reduce margins significantly more and hence it is reasonable to use imaging more sparsely in that case. Instead, in daily image guided radiotherapy (IGRT) the use of gold seed fiducials for the prostate bed localization is more beneficial. The random error of the PBM can be partially eliminated by the implantation of gold seed fiducials.

Surgical clips have also been used for the localization of the prostate bed in some reports. Sandhu et al. [5] used clips and orthogonal $\mathrm{kV}$ images to focus the treatment to the prostate bed. According to their results the PBM was slightly larger than in our investigation. Xu et al. [13] and Huang et al. [14] used the clips and $\mathrm{CBCT}$ and in their study the results for the PBM were close to ours. The clips were also seen in our study but we found it difficult to match according to clips because of their varying number and asymmetric shape.

We did not measure the intrafraction PBM but Klayton et al. [15] used Calypso and radiofrequency transponders to investigate the PBM during IMRT. They found out that PBM during the treatment fraction was likely to be the consequence of rectum and bladder filling like in a case of intact prostate motion which has been studied earlier [20-23]. In our clinic the volume of bladder is tried to be kept constant by the instructions for emptying the bladder and drinking a known amount of water before treatment. The volume of rectum instead is more difficult to control and therefore here the main reason for the PBM is varying filling of rectum. Huang et al. [14] calculated that the margins for the PBM during the treatment were $2.8,3.9$ and $4.3 \mathrm{~mm}$ in the LR, SI and AP axes, respectively. According to these results, our margins, which are needed for the situation where IGRT is performed on the first three treatment fractions and the rest of the fractions are given along with the offset SM, would decrease by $40 \%$ and $35 \%$ in the SI and AP axes, respectively. The margin in the LR direction is $18 \%$ larger in intrafractional analysis.

In conclusion, the linear PBM is largest in the SI and AP axis while rotation is largest in the sagittal plane. An automatic soft

Table 3

Systematic and random errors and CTV-PTV margins for the different localization methods [mm]

\begin{tabular}{|c|c|c|c|c|c|c|c|c|c|c|c|c|}
\hline & \multicolumn{3}{|c|}{ TPE/SM } & \multicolumn{3}{|c|}{ SUE/BL\&SM } & \multicolumn{3}{|c|}{$\mathrm{PBM} / \mathrm{BL}$} & \multicolumn{3}{|c|}{ Daily IGRT (data from Huang et al. [14]) } \\
\hline & LR & SI & AP & LR & SI & $\mathrm{AP}$ & LR & SI & $\mathrm{AP}$ & LR & SI & $\mathrm{AP}$ \\
\hline$\Sigma$ & 1.9 & 2.9 & 2.3 & 1.9 & 2.1 & 1.2 & 0.5 & 2.1 & 1.9 & 0.7 & 1.0 & 1.1 \\
\hline$\sigma$ & 1.9 & 3.2 & 4.2 & 1.9 & 2.2 & 2.0 & 0.5 & 2.5 & 3.2 & 2.0 & 2.8 & 3.1 \\
\hline Margin & ${ }^{\mathrm{a}} 4.9$ & 8.0 & 7.4 & ${ }^{\mathrm{b}} 2.4$ & 6.5 & 6.6 & ${ }^{\mathrm{a}} 1.4$ & 5.9 & 5.9 & 2.8 & 3.9 & 4.3 \\
\hline
\end{tabular}

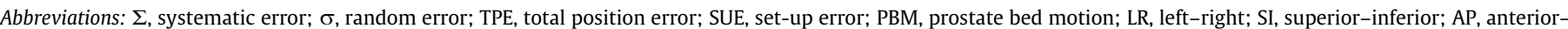
posterior; SM, skin marks; BL\&SM, bone localization and skin marks; BL, bone localization; IGRT, image-guided radiotherapy.

a Calculated by the Eq. (1).

b Calculated by the Eq. (2). 
tissue registration and gold seed fiducial localization do not give the same results for the PBM. The $\mathrm{BL}$ on the first three treatment fractions can reduce PTV margins by $52 \%, 18 \%$ and $10 \%$ in the LR, SI and AP axes, respectively, when compared to localization by the SM. Daily BL does not reduce margins significantly more and thus the gold seed fiducial localization in daily IGRT seems to be more reasonable.

\section{Conflict of interest}

There were no financial or personal relationships with other people or organizations that could inappropriately influence this work.

\section{References}

[1] Tsien C, Griffith KA, Sandler HM, et al. Long term results of three-dimensional conformal adjuvant and salvage radiotherapy after radical prostatectomy. Urology 2003;62:93-8.

[2] Bolla M, van Poppel H, Collette L, et al. Postoperative radiotherapy after radical prostatectomy; a randomised controlled trial (EORTC trial 22911). Lancet 2005;366:572-8.

[3] Neuhof D, Hentschel T, Bischof M, Sroka-Perez G, Hohenfellner M, Debus J. Long-term results and predictive factors of three-dimensional conformal salvage radiotherapy for biochemical relapse after prostatectomy. Int J Radiat Oncol Biol Phys 2007;67:1411-7.

[4] Schiffner DC, Gottschalk AR, Lometti M, et al. Daily electronic portal imaging of implanted gold seed fiducials in patients undergoing radiotherapy after radical prostatectomy. Int J Radiat Oncol Biol Phys 2007;67:610-9.

[5] Sandhu A, Sethi R, Rice R, et al. Prostate bed localization with image-guided approach using on-board imaging: reporting acute toxicity and implication for radiation therapy planning following prostatectomy. Radiother Oncol 2008;88:20-5.

[6] Ost P, De Meerleer G, De Gersem W, Impens A, De Neve W. Analysis of prostate bed motion using daily cone-beam computed tomography during postprostatectomy radiotherapy. Int J Radiat Oncol Biol Phys 2011;79:188-94.

[7] Teh BS, Mai WY, Augspurger ME, et al. Intensity-modulated radiation therapy (IMRT) following prostatectomy: more favorable acute genitourinary toxicity profile compared to primary IMRT for prostate cancer. Int J Radiat Oncol Biol Phys 2001;49:465-72.
[8] Cozzarini C, Fiorino C, Ceresoli GL, et al. Significant correlation between rectal DVH and late bleeding in patients treated after radical prostatectomy with conformal or conventional radiotherapy (66.6-70.2 Gy). Int J Radiat Oncol Biol Phys 2003;55:688-94.

[9] Williams C, Costa J, Mandia S, Henderson R, Marino R, Mendenhall N. Urethral catheterization facilitates preradiation fiducial marker placement in postprostatectomy patients. J Endourol 2012;26:467-8.

[10] Chinnaiyan P, Tomée W, Patel R, Chappell R, Ritter M. 3D-ultrasound guided radiation therapy in the postprostatectomy setting. Technol Cancer Res Treat 2003;2:455-8

[11] Paskalev K, Feigenberg S, Jacob R, et al. Target localization for postprostatectomy patients using CT and ultrasound image guidance. J Appl Clin Med Phys 2005;6:40-9.

[12] Cavalieri R, Gay H, Liu J, et al. Total error shift patterns for daily CT on rails image-guided radiotherapy to the prostate bed. Radiat Oncol 2011;6:142.

[13] Xu Z, Li T, Lee W, Hood R, Godfrey D, Wu G. SU-E-J-16: prostate bed motion during post-prostatectomy radiotherapy. Med Phys 2012;39:3655.

[14] Huang K, Palma D, Scott D, et al. Inter- and intrafraction uncertainty in prostate bed image-guided radiotherapy. Int J Radiat Oncol Biol Phys 2012:84:402-7.

[15] Klayton T, Price R, Buyyounouski MK, et al. Prostate bed motion during intensity-modulated radiotherapy treatment. Int J Radiat Oncol Biol Phys 2012;84:130-6.

[16] van Herk M, Remeijer P, Rasch C, Lebesque J. The probability of correct target dosage: dose-population histograms for deriving treatment margins in radiotherapy. Int J Radiat Oncol Biol Phys 2000;47:1121-35.

[17] Gill S, Pham D, Dang K, et al. Plan of the day selection for online image-guided adaptive post-prostatectomy radiotherapy. Radiother Oncol 2013;107:165-70.

[18] Connolly JA, Shinohara K, Presti Jr JC, Carroll PR. Local recurrence after radical prostatectomy: characteristics in size, location, and relationship to prostatespecific antigen and surgical margins. Urology 1996;47:225-31.

[19] Parker C, Warde P, Catton C. Salvage radiotherapy for PSA failure after radical prostatectomy. Radiother Oncol 2001;61:107-16.

[20] van Herk M, Bruce A, Kroes A, Shouman T, Touw A, Lebesque JV. Quantification of organ motion during conformal radiotherapy of the prostate by threedimensional image registration. Int J Radiat Oncol Biol Phys 1995:33:1311-20.

[21] Beard CJ, Kijewski P, Bussiere M, et al. Analysis of prostate and seminal vesicle motion: Implications for treatment planning. Int J Radiat Oncol Biol Phys 1996;34:451-8.

[22] Antolak JA, Rosen II, Chilldress CH, Zagars GK, Pollack A. Prostate target volume variations during a course of radiotherapy. Int J Radiat Oncol Biol Phys 1998;42:661-72.

[23] Nichol AM, Brock KK, Lockwood GA, et al. A magnetic resonance imaging study of prostate deformation relative to implanted gold fiducial markers. Int J Radiat Oncol Biol Phys 2007;67:48-56. 